\title{
Light Induced Modification of Graphene Oxide Layers on GaN Basis
}

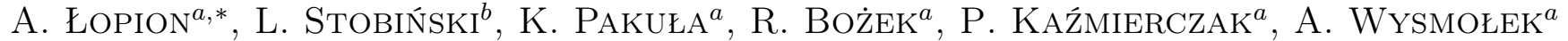 \\ AND R. STĘPNIEWSKI ${ }^{a}$ \\ ${ }^{a}$ Faculty of Physics, University of Warsaw, L. Pasteura 5, 02-093 Warsaw, Poland \\ ${ }^{b}$ Faculty of Chemical and Process Engineering, Warsaw University of Technology, \\ L. Waryńskiego 1, 00-645 Warsaw, Poland
}

\begin{abstract}
Graphene oxide suspension in various solvents was spin coated on metal organic vapor phase epitaxy grown $\mathrm{GaN}$ /saphire layers. Samples were characterised using the Raman spectroscopy and atomic force microscopy, before and after high temperature treatment. We found that graphene oxide was modifed by high temperature treatment, however a considerable modification was also observed as a result of impinged laser light incident due to the measurements. The Raman spectra were decomposed into two contributions showing different behaviour during the Raman scattering measurements.
\end{abstract}

DOI: 10.12693/APhysPolA.130.1169

PACS/topics: 73.22.-f, 78.55.-m, 78.30.-j, 07.05.Kf

\section{Introduction}

Graphene-based heterostructures are believed to make a vital contribution to future technological applications, in particular, employing its transparency and flexibility. Graphene oxide is also reported as a possible candidate for those applications [1]. Moreover, one of the advantages of graphene and graphene oxide is their biocompatibility, which makes graphene safe in biological and medical uses.

The term "graphene oxide" (GO) covers a whole group of nanomaterials derivable from graphite using various methodes. GO is a compound of carbon, oxygen and hydrogen, in variable proportions. The main property of graphene oxide is presence of graphene-like carbon rings, as well as a vast range of oxygen functional groups such as hydroxyl, carboxyl, epoxy, carbonyl, ester and others $(\approx 50 \mathrm{wt} \%$ of oxygen). Information about the actual GO composition, which is important from the point of view of applications, can be obtained from the Raman spectroscopy but diversity of this material allows only qualitative characterisation.

A novel, perspective device would be a hybrid consisting of a high-electron-mobility transistor (HEMT) with a graphene gate, which could be chemically functionalised for various chemical or biological applications. Such a gate can be alternatively made of GO, which in contrast to graphene can be more easily functionalised by exchanging the functional groups present in GO into different chemical moieties with the desired properties.

Considering its high tolerance to temperature and chemical environment, we chose $\mathrm{AlGaN} / \mathrm{GaN}$ junction as a good HEMT material candidate. Here, we report

*corresponding author; e-mail: aleksandra.lopion@gmail.com initial investigations of graphene oxide films, deposited on $\mathrm{GaN}$ layer, which could be employed in the proposed device.

\section{Experimental}

The substrate for the samples was metal organic vapour phase epitaxy (MOVPE) GaN layer grown on [0001] sapphire substrate with low temperature AlN buffer. The layer was $1100 \mathrm{~nm}$ thick and had smooth morphology with dislocation density about $10^{9} \mathrm{~cm}^{-2}$. Its electron concentration was below $10^{16} \mathrm{~cm}^{-3}$.

GO flakes were obtained by oxidation of graphite powder as per the modified Hummers method [2,3]. Light to dark yellow-brown GO is highly hydrophilic and forms stable aqueous suspension. Water suspension of GO flakes $(0.5 \%$ mass percentage) was diluted with methyl alcohol or ethyl alcohol [4] to improve wetting of hydrophobic gallium nitride substrate. The best result was obtained with 1:1 mixture of GO water suspension to ethyl alcohol (99.6\% purity); in such conditions, the GO flakes were most easily distributed and yielded smooth, vast surfaces. Homogeneity of the suspension was improved using ultrasonication (about $15 \mathrm{~min}$ ). Then, GO suspension was distributed by spin-coating on GaN substrate. In the next step of the preparation process, the samples were dried on a hot plate for $5 \mathrm{~min}$. The temperature of the hot plate was below boiling temperature of the applied solvent to evaporate dispersion medium without destroying the GO layer by rapid boiling process. Some samples were also heated for $10 \mathrm{~min}$ on a hot plate at temperatures up to $300^{\circ} \mathrm{C}$. During such high temperature treatment, notable change of colour of GO layers, from light brown to black, was observed. We assigned these changes to physical processes of release of the bound solvent and chemical modification of GO. It is reported that partial reduction of GO occurs at temperatures around $240^{\circ} \mathrm{C}[5]$ and that process is observed 
here. Thus, there are two types of investigated samples: the first one - GO layers on GaN substrate, and the second - GO layers treated with high temperature and partially reduced to RGO.

The samples were characterised using atomic force microscopy (AFM) and the Raman spectroscopy. The AFM measurements were performed in the tapping mode using the MultiMode AFM with a Nanoscope IIIa controller (former Digital Instruments) to investigate the morphology of the GO layer and to estimate its thickness. The Raman spectroscopy was performed at laser excitation wavelength of $532 \mathrm{~nm}$. Several spectra where collected one after another at the same place of the sample with the same illumination time to monitor the influence of the excitation light.

\section{Results and discussion}

\subsection{AFM studies}

AFM measurements showed that the investigated layers were fairly homogeneous. Their characteristic feature was high density of wrinkles, Fig. 1, similar to those found on graphene multilayers [6]. Those wrinkles had mainly a random orientation. In some cases, they originated radially from a hillock. Their typical dimensions were: length $<10 \mu \mathrm{m}$, height $<20 \mathrm{~nm}$ and width in the range from 100 to $200 \mathrm{~nm}$. Thickness of the graphene oxide layer was estimated by cutting of the layer with a sharp steel blade, which left the gallium nitride layer beneath GO undamaged. The profile measurements were performed evenly along a scratch with a length of a few millimetres. The thickness of the layer remained close to $20 \mathrm{~nm}$. Slightly thicker areas could be correlated with groups of wrinkles.

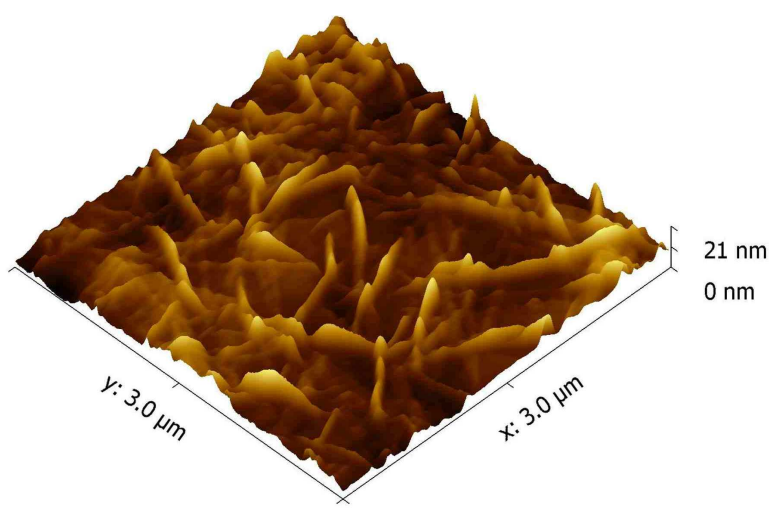

Fig. 1. Typical morphology of the investigated samples, AFM.

\subsection{Raman spectroscopy results}

The main property of the group of materials called graphene oxide is presence of graphene-like carbon rings. Thus, in the Raman spectra of GO one can distinguish $G$ peak, similar to that of graphene, corresponding to hexagonal crystal structure normal mode at about $1600 \mathrm{~cm}^{-1}$. Furthermore, $D$ peak (at about $1350 \mathrm{~cm}^{-1}$ ) corresponding to high density phonon states in point $K$ of the Brillouin zone, permitted by defects, in GO has intensity comparable to $G$ peak [7]. That is the result of presence of various functional groups intruding periodicity of GO crystal structure. Higher order peaks of $D$ and $G$ transitions are also present, centred at $2700 \mathrm{~cm}^{-1}$ and $2950 \mathrm{~cm}^{-1}$, which was observed for GO films before [8]. The Raman spectra of our high temperature treated layers (Fig. 2A), apart from the presence of a peak centred at about $3180 \mathrm{~cm}^{-1}$, were similar to the majority of GO Raman spectra.

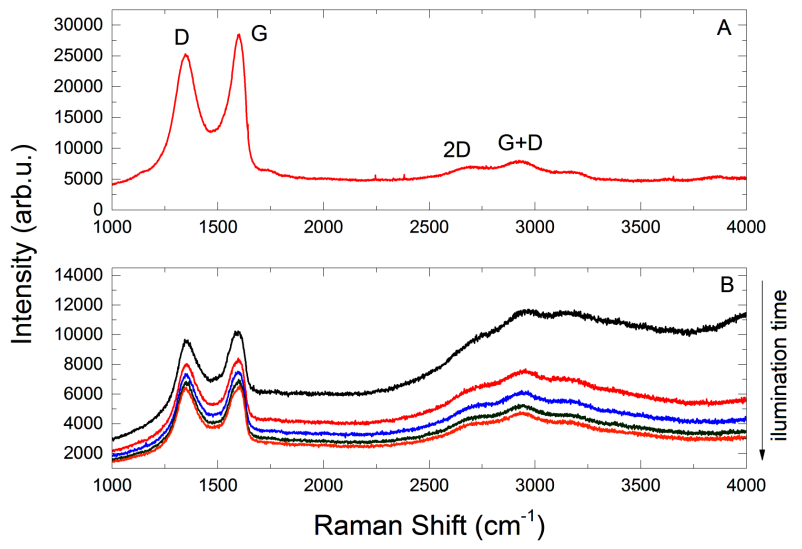

Fig. 2. (A) Exemplary spectrum of high temperature treated layer, (B) successive Raman-luminescence spectra of not heated GO layer.

Samples not heated above $80^{\circ} \mathrm{C}$ gave different spectra. We observed another contribution overlapping a typical graphene oxide spectrum (Fig. 2B). We found out that the second component changed during the Raman scattering measurements as a result of laser light. After subsequent periods of illumination, the second component gradually decreased and finally spectra similar to those for high temperature treated samples were observed. Taking into account the observed dependence of the wide band part of the spectrum on the excitation energy (not shown), we assumed that it is related to luminescence.

To resolve these Raman-luminescence combined spectra, we developed a very effective procedure based on principal component analysis (PCA) [9] and the Hilbert space functional analysis. This procedure allows separating different effects without any direct fitting. The main advantage of the procedure is the possibility to reduce the dimensionality of the experimental data set and to quickly identify defected spectra in the set. Within PCA, it is possible to simply determine the number of the required "principal components". In our case of optical spectra, each spectrum can be reconstructed using only two functions (basis) and their corresponding decomposition coefficients. However, to provide physical interpretation of the obtained results, it is necessary to choose an appropriate basis that corresponds to physical processes 


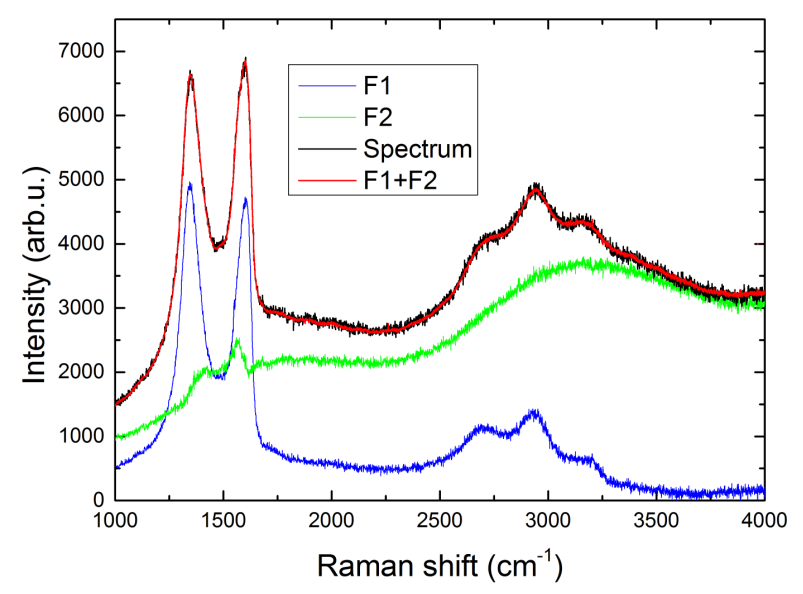

Fig. 3. Exemplary decompositon of Raman spectra into F1 - "Raman" and F2 - "luminescence" parts.

responsible for the observed spectra. We accomplished it by applying Hilbert space analysis, and rotating the basis received within PCA. We chose two different functions (vectors in Hilbert space), one called "Raman" and another, called "luminescence". The "Raman" part was chosen to be similar to the Raman spectra of high temperature treated samples. The "luminescence" contribution was chosen as a wide background band, for which the influence of the Raman $G$ and $D$ lines reaches the minimum. Example of decomposition of the original spectrum into these components is shown in Fig. 3. The intensity changes of the "Raman" and "luminescence" components as a function of accumulated time of illumination are shown in Fig. 4.

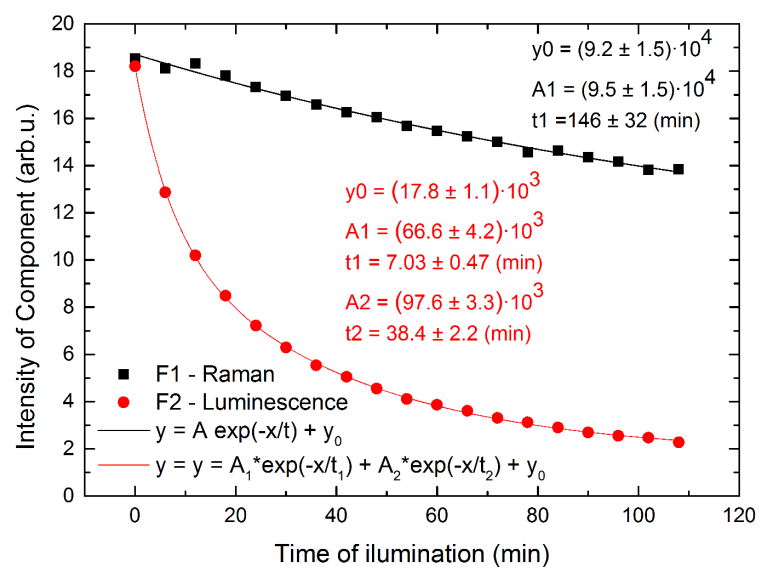

Fig. 4. Points - components intensity as a function of accumulated time of illumination, lines - fitting with exponential and biexponential decay for "Raman" and "luminescence" parts, respectively.

We interpret the moderate exponential decrease of the "Raman" part as a result of partial GO reduction [10]. Interpretations of biexponential decay of the "luminescence" part is more complicated. First of all, we assume that despite the process of sample drying, there is still water/alcohol bound in GO layer [11]. Its removal yields background decay. The two different decay constants (ratio of about 5-6) can be related to apparently different activation energies responsible for this process. It could be caused by two modes of bound dispersion media - water or alcohol. Moreover, dispersion medium is removed both, from the surface and from the volume which requires diffusion to the surface, which could result in biexponential decay. It is reported that heating GO layers results in the change of optical properties [11], which can be related to the observed disappearance of luminescence. Tentative interpretation presented above requires verification.

\section{Conclusions}

Graphene oxide layers were successively deposited on $\mathrm{GaN} /$ sapphire substrate, which allows further studies of HEMT structures with functionalised GO gate. In the Raman spectrum of virgin samples, two different components were observed, one corresponding to phonon excitations of GO, and the second related to photoluminescence of yet unknown origin. These components were successively separated using principal components analysis and the Hilbert space analysis methods. The luminescence part of the spectrum disappeared after temperature treatment at $300^{\circ} \mathrm{C}$, and shows biexponential decay under illumination of light in visible range.

\section{References}

[1] H.A. Becerril, J. Mao, Z. Liu, R.M. Stoltenberg, Z. Bao, Y. Chen, ACS Nano 2, 463 (2008).

[2] W.S. Hummers, R.E. Offeman, J. Am. Chem. Soc. 80, 1339 (1958).

[3] L. Stobinski, B. Lesiak, A. Malolepszy, M. Mazurkiewicz, B. Mierzwa, J. Zemek, P. Jiricek, I. Bieloshapka, J. Electron Spectrosc. 195, 145 (2014).

[4] J.I. Paredes, S. Villar-Rodil, A. Martinez-Alonso, J.M.D. Tascon, Langmuir 24, 10560 (2008).

[5] H.K. Jeong, Y.P. Lee, M.H. Jin, E.S. Kim, J.J. Bae, Y.H. Lee, Chem. Phys. Lett. 470, 255 (2009).

[6] J. Borysiuk, R. Bożek, K. Grodecki, A. Wysmołek, W. Strupiński, R. Stępniewski, J.M. Baranowski, J. Appl. Phys. 108, 013518 (2010).

[7] T.V. Cuong, V.H. Pham, Q.T. Tran, S.H. Hahn, J.S. Chung, E.W. Shin, E.J. Kim, Mater. Lett. 64, 399 (2010).

[8] K.N. Kudin, B. Ozbas, H.C. Schniepp, R.K. Prud'homme, I.A. Aksay, R. Car, Nano Lett. 1, 36 (2008).

[9] I. Jolliffe, Principal Component Analysis, 2nd ed., Springer Series in Statistics, Springer, New York 2002.

[10] S. Claramunt, A. Varea, D. Lopez-Diaz, M.M. Velázquez, A. Cornet, A. Cirera, J. Phys. Chem. C 119, 10123 (2015).

[11] M. Ghosh, L. Pradipkanti, V. Rai, D.K. Satapathy, P. Vayalamkuzhi, M. Jaiswal, Appl. Phys. Lett. 106, 241902 (2015). 